\title{
CARACTERIZAÇÃO MORFOLÓGICA DE UM TRECHO DO RIO ITAPOCU, REGIÃO NORTE DE SANTA CATARINA: SUBSÍDIOS PARA A IDENTIFICAÇÃO DE ÁREAS DE RISCO GEOMÓRFICO

\author{
Fábio Pinto ${ }^{1}$, Débora Brentano ${ }^{2,1}$ and Fernanda Simoni ${ }^{2}$
}

\begin{abstract}
Processos morfodinâmicos desenvolvidos em ambiente fluvial, estão relacionados a fatores naturais que propiciam alterações periódicas na paisagem e às litoestruturas preexistentes que controlam o substrato do vale fluvial. Os principais fatores naturais que propiciam as alterações, correspondem a atividade tectônica, capaz de alterar os padrões altimétricos do terreno, e a atividade climática, controladora do regime de precipitação. Por outro lado, a depender da composição e da configuração estrutural do substrato, este poderá controlar a mecânica evolutiva dos rios. Estudos de sensitividade fluvial, podem se restringir a análises encerradas em processos ou parâmetros particulares, com a finalidade de elucidar problemas específicos do ambiente. Por outro lado, podem possuir tendências generalistas, principalmente quando centrados na discussão dos efeitos sinergísticos dos processos que imputam alterações ao ambiente e dos controles naturais que proporcionam resistência e resiliência ao ambiente. Desse modo, o trabalho pretendido, visa analisar as interações entre chuvas e condicionantes do substrato na evolução da paisagem fluvial, através da integração de dados pluviométricos com dados geoespaciais, extraídos de mapeamento multitemporal das alterações morfológicas nas geoformas do vale fluvial.
\end{abstract}

Palavras-chave:

\section{Keywords}

morfologia fluvial, sensitividade de paisagem, cartografia temática. 


\section{INTRODUÇÃO}

A sensitividade denota a paisagem como o produto de um conjunto de fenômenos que organizam e retrabalham as geoformas da paisagem, obedecendo às características do substrato (Fryirs, 2017) ou especificamente ao arcabouço litoestrutural de controle do terreno.

A análise da sensitividade, implica na avaliação da paisagem a partir da investigação dos seus limiares de estabilidade (geomorphic thresholds), propriamente da identificação dos fatores de modificação e dos controles geomórficos do terreno, permitindo a elaboração de modelos geoambientais fidedignos para o desenvolvimento da política ambiental, o planejamento da ocupação territorial, a melhoria da qualidade funcional do ambiente e, consequentemente, para a gestão ambiental e de riscos naturais (SCHUMM, 1979; SILVA \& SILVA, 2016; FRYIRS, 2017) (Fryirs, 2017; Schumm, 1979).

Geoformas sedimentares associadas às paisagens fluviais, são especialmente sensíveis a quaisquer perturbações nas condicionantes ambientais do sistema (SCHUMM, 1979, 1993; HOLBROOK \& SCHUMM, 1998; ) (Crosby \& Whipple, 2006). Condições de desequilíbrio, com alterações nos padrões de migração lateral, incisão, agradação e degradação sedimentar, e mudanças substanciais na paisagem fluvial, decorrem por influência de processos que podem provocar alterações nos níveis de base locais, ou fluviais, como aqueles associados ao clima (SCHUMM, 1979, 1993; ) (Crosby \& Whipple, 2006).

A precipitação é considerada o principal agente de modificação da paisagem sob controle do clima, com impacto direto no regime de escoamento superficial, sendo este, um dos mecanismos de modelação mais relevantes (GILBERT, 1877; ) (Deal, Botter, \& Braun, 2018; Scheidegger \& Hydrogeomorphology, 1973). Entretanto, há uma disparidade na influência da precipitação na paisagem (Deal et al., 2018). Processos morfodinâmicos são influenciados pelo montante anual da precipitação, mas são os extremos episódicos que, em curtos períodos de tempo, afetam intensamente a paisagem (GILBERT, 1877; Basso, Frascati, Marani, Schirmer, \& Botter, 2015; Scheidegger \& Hydrogeomorphology, 1973).

Portanto e considerando que o reconhecimento da sensitividade, proporciona a identificação dos controles litoestruturais que imputam resistência geomórfica ao sistema; que a integração de dados relativos a esses controles, com dados de origem climática, como aqueles relativos a extremos de precipitação, auxilia na investigação dos limiares de estabilidade do ambiente; que um distúrbio que ultrapassa o limiar de estabilidade do ambiente, tem propensão para ocasionar alterações reais e duradouras, tanto no sentido de migração lateral, de incisão, ou alterações nas dimensões do canal fluvial; e, finalmente, que os resultados da análise de sensitividade, podem ser traduzidos em produtos de cartografia temática, úteis para a gestão e o planejamento territorial, o trabalho analisará as interações entre chuvas e condicionantes do substrato na evolução das paisagens fluviais.

\footnotetext{
${ }^{1}$ Mestrado Profissional em Clima e Meio Ambiente, Florianópolis, Santa Catarina, Brasil

2 Instituto Federal de Santa Catarina, 3) Eng. Civil, Dra. Coorientadora

Emails: brentano@ifsc.edu.br (Débora Brentano), fernanda@ifsc.edu.br (Fernanda Simoni)
}

\section{Corresponding author:}

Fábio Pinto, Mestrado Profissional em Clima e Meio Ambiente, Florianópolis, Santa Catarina, Brasil

Email: fabiomacielpinto@gmail.com 
Para tanto, utilizará como área de estudo, um trecho do rio Itapocu, situado entre os municípios de Corupá, Jaraguá do Sul, Guaramirim e Araquari, no Estado de Santa Catarina. Nesse trecho, será determinada a sensitividade do ambiente fluvial ao desenvolvimento de processos morfodinâmicos capazes de ocasionar alterações morfológicas cartografáveis, nos padrões de estilo fluvial. Para isso, almeja-se caracterizar ajustes e alterações espaciais na morfologia do canal do rio; caracterizar litoestruturas associadas ao controle morfoestrutural do canal; analisar a relação entre episódios de extremos de precipitação e alterações espaciais na morfologia do canal. Ao final será elaborada carta de sensitividade ao desenvolvimento de processos de ajuste morfológico, ou migração do canal fluvial.

\section{REVISÃO BIBLIOGRÁFICA}

Gilbert (1877), identificou agentes de esculpimento e degradação da paisagem, elencando a chuva dentre os principais. Para Gilbert (1877), todos os processos de denudação são diretamente afetados pelo montante médio das chuvas ao longo do ano, sendo acelerados ou diminuídos pelas variações periódicas nas taxas de precipitação. Para os rios, os períodos em que a precipitação provoca eventos episódicos de cheias e inundações, são mais significativos para os processos de modelagem da paisagem fluvial do que períodos de monotonia, ou de chuvas bem distribuídas (GILBERT, 1877).

Scheidegger and Hydrogeomorphology (1973), em premissa semelhante, considera a água, o agente mais importante na modelagem da paisagem, causando alterações no equilíbrio das formas de relevo. A água de runoff ou de escoamento superficial, causaria os principais efeitos de modelação, afetando a estabilidade das encostas, parcialmente percolando no substrato, alimentando os aquíferos e, parcialmente, sendo capturada pelos rios (Deal et al., 2018; Scheidegger \& Hydrogeomorphology, 1973).

Nos rios, eventos hidrológicos significantes que incrementam o fluxo hídrico por períodos curtos de tempo, são relativamente mais significativos para a modelagem da paisagem, ante as variações médias no montante da precipitação (GILBERT, 1877; ) (Scheidegger \& Hydrogeomorphology, 1973). Esses eventos estão diretamente associados à chuva dramaticamente concentrada que ocasiona o aumento da capacidade erosiva, ao ponto de proporcionar alterações morfológicas drásticas no sistema fluvial, causando destabilizações relativas ao rompimento de margens, além de enchentes (GILBERT, 1877; ) (Scheidegger \& Hydrogeomorphology, 1973).

Nesse sentido, Bower, Hannah, and Mcgregor (2004), estabeleceram equações que correlacionam fluxo hídrico, precipitação, temperatura do ar e as alimentaram com dados coletados durante 25 anos, relativos a esses parâmetros. Demonstraram que rios são sensíveis às variações nos padrões das chuvas e, enquanto não foi estabelecida relação clara entre temperatura do ar e fluxo hídrico (Bower et al., 2004).

Para Basso et al. (2015), a pluviosidade afeta os ambientes fluviais de maneira não linear, a depender do contexto climático, ao produzir regimes heterogêneos, ou monótonos de escoamento nos rios. Situações em que o contexto é propício à concentração de chuvas em determinados períodos, favorecem a erosão e o transporte clástico, sendo mais significativos para a evolução paisagem fluvial.

Desse modo, há uma disparidade ante a escala geológica do tempo na ação climática em diferentes paisagens pois, enquanto as taxas de chuva associadas ao ambiente fluvial, em horas ou dias, podem conduzir a respostas erosivas relevantes e registráveis, por outro lado, em outros ambientes da paisagem, uma resposta relevante sob as mesmas taxas, poderá levar séculos, ou milênios (Deal et al., 2018).

A influência de mudanças climáticas, igualmente, ocorrerá de maneira não linear, podendo impactar na disponibilidade de sedimentos, modificar os regimes de escoamento, reduzir ou ampliar a energia do 
fluxo hídrico (Deal et al., 2018). Prudhomme et al. (2013a, 2013b), de maneira generalizada, consideram bacias hidrográficas sensíveis a mudanças climáticas. Ainda que cada bacia absorva e responda de forma particular aos impactos das mudanças, obedecendo às suas características físicas, respostas geomórficas a eventos extremos, ocasionados por alterações nos padrões do clima, podem ocorrer em período, relativamente, curto ( 2 a 20 anos).

Nesse contexto, cenários de mudanças climáticas com aumento na concentração de $\mathrm{CO} 2$ e aerossóis de sulfato na atmosfera, são especialmente preocupantes quanto ao impacto nas bacias e rede hidrográfica. A depender do contexto, essas condições podem ocasionar aumento nas taxas de precipitação e no volume de água transportada nos rios, com alterações temporais nos períodos de ocorrência de inundações e de pico de inundações (Jha, Arnold, Gassman, Giorgi, \& Gu, 2006; Simonovic $\& \mathrm{Li}, 2004)$.

Para N Notebaert and Verstraeten (2010), em análise de escala geocronológica, os efeitos de variações nos padrões climáticos em ambientes fluviais são claros. Dados estratigráficos, radiométricos e paleoclimáticos, extraídos de amostras de sedimentos de planícies fluviais da região central e leste da Europa, denotam clara relação entre alterações nos padrões de deposição aluvial e mudanças no clima ocorridas ao longo do Holoceno, com mudanças no clima impactando diretamente nos padrões de escoamento e deposição dos rios (Notebaert \& Verstraeten, 2010).

\section{METODOLOGIA}

A metodologia se baseia na integração de dados morfoestruturais e pluviométricos, estes visando a identificação de episódios de extremos para incorporação na análise da sensitividade. Uma vez identificados os episódios.

Imagens de satélite serão utilizadas para caracterizar a rede fluvial em período diretamente associado aos eventos pluviométricos identificados. A partir das imagens serão obtidos dados geoespaciais (mapeamentos das margens fluviais e geoformas de fundo de vale para diferentes datas), buscando-se a identificação de possíveis alterações ocorridas após a ocorrência de eventos extremos.

\section{ÁREA DE ESTUDO}

Para mapear a sensitividade do canal fluvial, adotou-se uma área de estudos corresponde a um trecho do rio Itapocu, localizado em Santa Catarina, entre os Municípios de Corupá, Jaraguá do Sul, Guaramirim e Araquari (Figura 1). $\mathrm{O}$ trecho abrange a um segmento longitudinal do rio, com cerca de $52,3 \mathrm{~km}$, limitado na montante pela confluência dos rios Novo e Humboldt (UTM 675.832 E / 7.075.311 S) e na jusante pela desembocadura do rio Putanga (UTM 712.028 E / 7.063.098 S).

\section{EVENTOS PLUVIOMÉTRICOS SELECIONADOS}

Dados horários brutos, sem correção de falhas, foram extraídos de pluviômetros automáticos da rede observacional do Centro Nacional de Monitoramento e Alertas de Desastres Naturais - CEMADEN (www2.cemaden.gov.br). Os dados foram obtidos para os municípios de Corupá, Jaraguá do Sul, Guaramirim e Araquari/SC, abrangendo o período entre 01/01/2016 e 31/08/2021 (2070 dias). Em seguida, houve a seleção primária das estações ativas, preferenciando-se aquelas situadas a uma distância de até $250 \mathrm{~m}$ da área de estudo. Ou seja, aproximadamente instaladas no vale fluvial e consideradas representativas para eventos incidentes na área. As estações selecionadas foram: João Tozini 


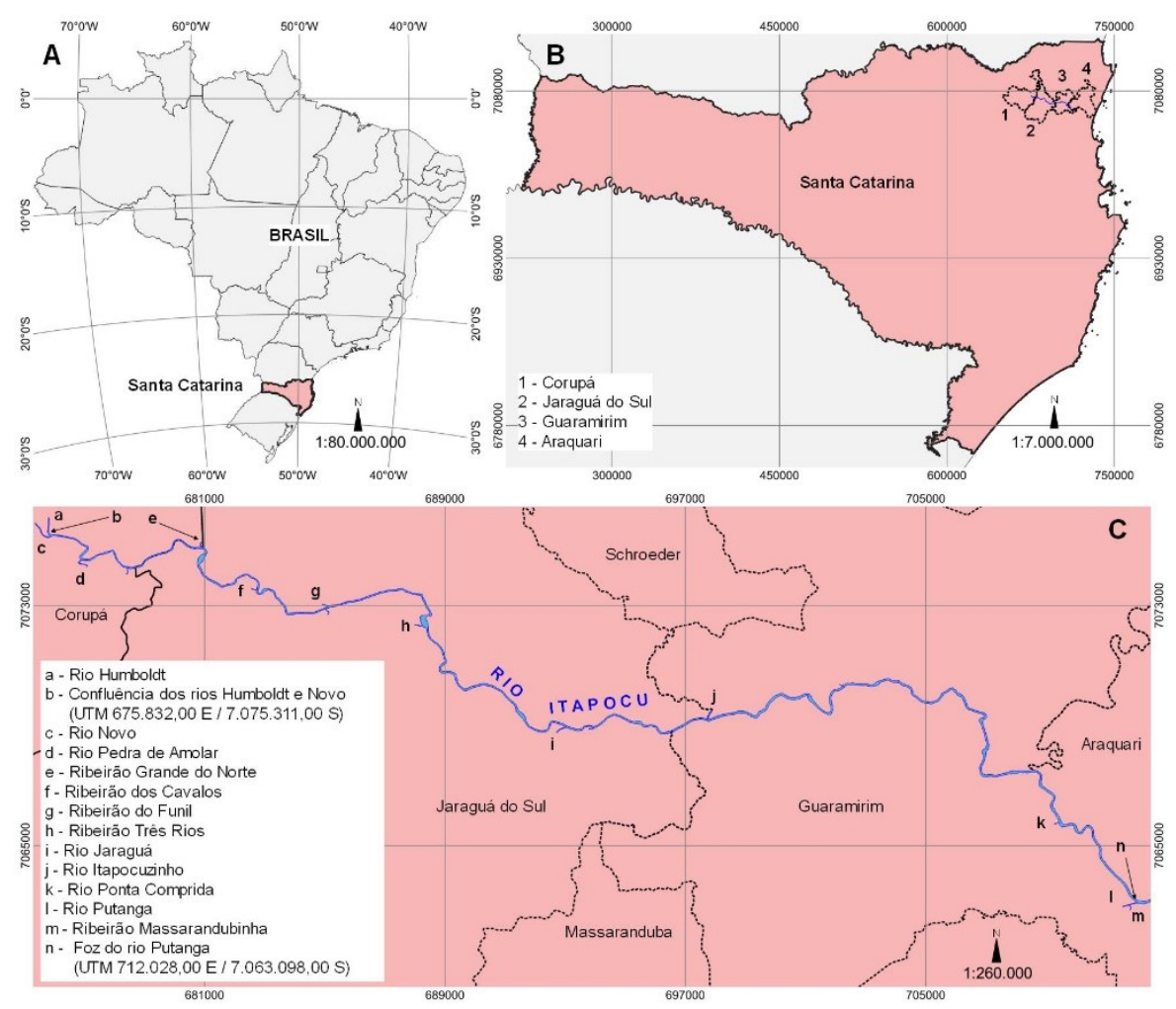

Figure 1. - Croqui de localização da área de estudo e de identificação de hidrônimos para referência situacional. Fonte de dados geoespaciais brutos: SDE (2015), IBGE (2020).

- 420450903A, situada em Corupá; Bombeiro do Centro - 420890601A e SAMAE Ilha da Figueira 420890606A, situadas em Jaraguá do Sul; e Bananal - 420650403A, situada em Guaramirim.

Essas estações apresentaram, respectivamente e aproximadamente $2,5 \% ; 0,8 \% ; 2 \%$; e $0,5 \%$ de falhas nos dados brutos do período. Desse modo, os eventos selecionados para análise, foram extraídos apartir da estação com menor quantidade de falhas (420650403A - Bananal). Foram obtidas as frequências acumuladas diárias $(24 \mathrm{~h})$ para a estação e elencadas aquelas superiores a $100 \mathrm{~mm}$, correspondentes aos dias 07/02/2020 (126,8mm), 19/05/2017 (119,8mm), 18/02/2019 (112,2mm), 22/05/2016(108mm), $27 / 11 / 2020(104,8 \mathrm{~mm})$ e $27 / 02 / 2021(101 \mathrm{~mm})$. 


\section{MAPEAMENTO MORFOESCULTURAL}

Os procedimentos serão desenvolvidos em ambiente GIS - QGIS3.20. O mapeamento será iniciado com o delineamento e classificação dos limites do fundo do vale, os quais restringem a evolução lateral do canal fluvial. Essa etapa será realizada através de fotointerpretação (Arcanjo \& Fotogeologia, 2011; Guy, 1966; Soares \& Fiori, 1976) e vetorização das margens, com o uso de Modelos Digitais de Terreno (MDT), resolução espacial (r.e.) $1 \mathrm{~m}$, do levantamento aerofotogramétrico de Santa Catarina, disponíveis na plataforma SIGSC (sig.sc.gov.br). Os MDTs serão renderizados em modelo de relevo sombreado de altitude $45^{\circ}$, azimute $315^{\circ}$ e fator $\mathrm{Z}$ de $3 \mathrm{~m}$.

Nas etapas de mapeamento multitemporal, dados geoespaciais serão extraídos de imagens multiespectrais, Level-1C, sensores MSI (Multispectral Instrument) da missão Sentinel-2. As imagens selecionadas, correspondem a cenas disponíveis nas plataformas Copernicus Open Acess Hub (scihub.copernicus.eu) e EarthExplorer (earthexplorer.usgs.gov), capturadas antes e depois dos eventos selecionados: 18/02/2019 (datas das imagens: 30/01 e 15/04/2019), 27/11/2020 (datas: 15/11 e 30/12/2020) e 27/02/2021 (datas: 23/02 e 10/03/2021).

A partir das imagens, serão realizadas classificações morfológicas multitemporiais e de estilos do canal ao longo do período de estudo, as quais seguirão as etapas: a) realce do canal através do índice da diferença normalizada da água de Mcfeeters (1996) [NDWI=B3(GREEN)B8(NIR)/B3(GREEN)+B8(NIR)]; b) fotointerpretação (Arcanjo \& Fotogeologia, 2011; Guy, 1966; Soares \& Fiori, 1976) e vetorização; c) segmentação do canal em zonas com padrões morfológicos homólogos (Arcanjo \& Fotogeologia, 2011); d) caracterização morfológica e morfométrica do canal (Brierley \& Fryirs, 2005; Fryirs, Wheaton, \& Brierley, 2016; Rinaldi, Gurnell, Tánago, Bussettini, \& Hendriks, 2016).

Finalmente, haverá a delimitação das geoformas, a qual visa a subdivisão do terreno em unidades morfológicas e a extração de informações multitemporais sobre a evolução espacial dessas unidades ao longo do período de estudo. Esse procedimento ocorrerá a partir do realce das geoformas através de razões de bandas, r.e. 10 e $20 \mathrm{~m}$, conforme Miranda, Duarte, Gomes, Souza, and Neto (2019), [B8(NIR)/B12(SWIR2)] e [B8a(VNIR)/B12(SWIR)], seguido de fotointerpretação, vetorização e classificação das geoformas de acordo com suas características morfológicas e estruturais, conforme convenções de IBGE (1998, 2009).

A partir da identificação de ocorrências, ou não, de alterações morfológicas no canal fluvial ao longo do período, após os eventos selecionados, será elaborado produto cartográfico final. Esse produto corresponderá a um mapa de sensitividade do ambiente fluvial, abrangendo a área de estudo, em escala de detalhe a semidetalhe. O mapa identificará as geoformas existentes no fundo do vale fluvial, o canal atual do rio e os trechos do rio e do vale sensíveis a alterações morfológicas balizadas pelo padrão dos eventos pluviométricos selecionados. Desse modo, o produto demonstrará os trechos sensíveis a alterações, tendo em vista a ocorrência de determinados fenômenos pluviométricos (com determinada intensidade e duração).

\section{REFERÊNCIAS}


Arcanjo, J. B. A., \& Fotogeologia. (2011).

Barros, A. C. M., Souza, J. O. P., \& Correa, A. C. B. (2010). Sensitividade da paisagem na bacia do riacho do mulungu. Belém de São Francisco, Pernambuco.

Basso, S., Frascati, A., Marani, M., Schirmer, M., \& Botter, G. (2015). Climatic and landscape controls on effective discharge. Geophysical Research Letters, 42, 8441-8447.

Bower, D., Hannah, D. M., \& Mcgregor, G. R. (2004). (Vol. 18).

Brierley, G. J., \& Fryirs, K. A. (2005). Geomorphology and river management: Applications of the river styles framework. Oxford, UK: Blackwell Publishing.

Brunsden, D., \& Thornes, J. B. (1979).

Crosby, B. T., \& Whipple, K. X. (2006). Knickpoint initiation and distribution within fluvial networks: 236 waterfalls in the. Waipaoa River, North Island, New Zealand. Geomorphology.

Deal, E., Botter, G., \& Braun, J. (2018). Understanding the role of rainfall and hydrology in determining fluvial erosion efficiency. Journal of Geophysical Research: Earth Surface(123), 744-778.

Estatística, I. I. B. D. G. E. (n.d.). Retrieved from <https://www.ibge.gov . br/>

Estatística, I. I. B. D. G. E. (1998).

Fryirs, K. A. (2017). River sensitivity: a lost foundation concept in fluvial geomorphology. Earth Surface Processes and Landforms, 42, 55-70.

Fryirs, K. A., Wheaton, J. M., \& Brierley, G. J. (2016).

Gilbert, G. K. (n.d.).

Guy, M. (1966). Quelques principes e quelques experiences sur la methodologie de la photointerpretation. In Symposium International de Photo-Interpretation (SIPI) (Vol. 2, p. 21-41).

Holbrook, J., \& Schumm, S. A. (1999).

(n.d.). Retrieved from $<$ http: //sigsc.sc.gov.br/>

Jha, M., Arnold, J. G., Gassman, P. W., Giorgi, F., \& Gu, R. R. (2006). Climate change sensitivity assessment on Upper Mississippi river basin streamflows usin SWAT. Journal of the american water resources association(4), 997-1015.

Mcfeeters, S. K. (1996). The use of the Normalized Difference Water Index (NDWI) in the delineation of open water features. International Journal of Remote Sensing(7), 1425-1432.

Miranda, M. P., Duarte, C. R., Gomes, D. D. M., Souza, C. D., \& Neto, C. A. S. (2019). Análise comparativa de imagens sentinel 2 a (msi) e landsat-8 (oli) aplicadas ao mapeamento geológico, região de itataia. Santa Quitéria, $\mathrm{CE}$.

Notebaert, B., \& Verstraeten, G. (2010). Sensitivity of west and central european river systems to environmental changes during the Holocen: a review. Earth-Science Reviews(103), 163-182.

Prudhomme, C., Crooks, S., Kay, A. L., \& Reunard, N. (2013a). Climate change and river flooding: parte 1 classifying the sensitivity of British catchments. Climatic Change, 119, 933-948.

Prudhomme, C., Crooks, S., Kay, A. L., \& Reunard, N. (2013b). Climate change and river flooding: parte 2 sensitivity characterisation for british catchments and example vulnerability assessments. Climatic Change, 119, 949-964.

Rinaldi, M., Gurnell, A. M., Tánago, M., Bussettini, M., \& Hendriks, D. (2016). Classification of river morphology and hydrology to support management and restoration. Aquatic Sciences, 78, 17-33.

Scheidegger, A. E., \& Hydrogeomorphology. (1973). Journal of Hydrology, 20, 193-215.

Schumm, S. A. (1979). 
Schumm, S. A. (1993). River response to baselevel change: implication for sequence stratigraphy. The Journal of Geology, 101(2), 279-294.

Simonovic, S. P., \& Li, L. (2004). (Vol. 18).

Soares, P. C., \& Fiori, A. P. (1976).

\section{Author Biographies}

Fábio Pinto Discente do Mestrado Profissional em Clima e Ambiente

Débora Brentano Orientadora, docente do Instituto Federal de Santa Catarina e do Mestrado Profissional em Clima e Ambiente.

Débora Brentano Co-orientadora e docente do Instituto Federal de Santa Catarina. 